Journal of Theoretical and Applied Mechanics, Sofia, 2016, vol. 46, No. 1, pp. 65-82

\title{
T-SHAPED FRAME CRITICAL AND POST-CRITICAL ANALYSIS
}

\author{
Albena Doicheva \\ Technical Mechanics Department, \\ University of Architecture Civil Engineering and Geodesy, \\ 1, Hristo Smirnenski Blvd, Sofia 1046, Bulgaria, \\ e-mails: doicheva_fhe@uacg.bg, a_doicheva@abv.bg
}

[Received 04 January 2016. Accepted 29 February 2016]

\begin{abstract}
The paper shows solution of a T-shaped frame, strengthened with two linear springs, regarding critical and post-critical analysis. The solution is exact using the Euler elastic approach and the frame of reference, originated in the point of column axis inflexion. The derived Numerical results show the effect of the springs strengthening for the critical and the post-critical system behaviour. The influence of the geometry change is analyzed, as well.

KEY WORDS: Post-buckling analysis, exact-solution, non-prismatic columns, elastic analogy rule.
\end{abstract}

\section{Introduction}

The critical and the post-critical analysis of deformable systems is an important problem in structural mechanics and one of the most important design components and verification procedures. The related literature is rich in solutions of typical examples, conclusions and recommendations, obtained by means of a wide variety of exact and approximate approaches in monographs [1-8], papers $[9,10]$ etc. The solutions obtained on the basis of the exact differential equation of the elastic lines of beam-columns are of utmost importance for both the reliable conclusions derived and the possibility to assess the accuracy of approximate approaches such as finite element and boundary methods, finite difference method, differentially quadrature methods, etc. The present paper deals with the critical and the post-critical analysis of a frame, proposed by Życzkowski [10]. The frame consists in a non-prismatic column OB and a transverse rigid beam $\mathrm{ABC}$ of length $2 b$, symmetrically attached to the column. Two linear elastic springs OA and OC connect the extremities of the beam with 
the clamped end $\mathrm{O}$ of the column (Fig. 1). A load $P$ of a constant magnitude, application point $\mathrm{B}$ and direction is applied at the upper end of the column. Życzkowski's solution is approximate, based on a perturbation technique and the precondition that the axial deformation of the column and the influence of the shear force on the elastic line are negligibly small. The same frame, but with prismatic column, is considered in the present paper. The solution is exact applying the idea of the Eulerian elastica [1, 2, 9, 11]. The axial deformation of the elastic line is disregarded at first. Its effect is accounted for, at the end of the paper, in order to find out how it affects the critical and the post-critical equilibrium system states.

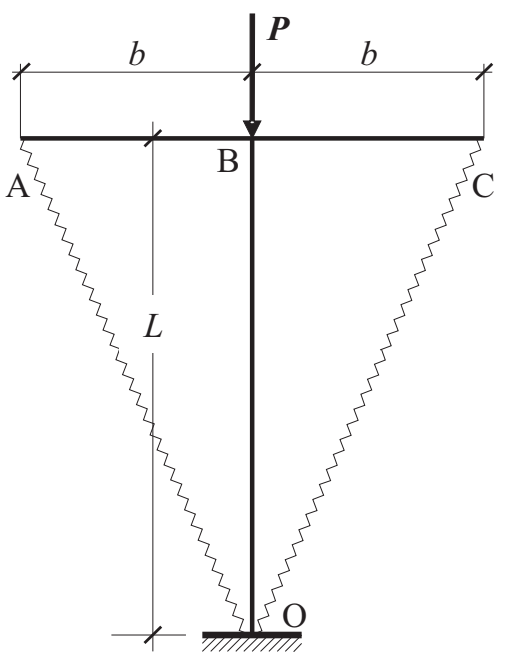

Fig. 1. Życzkowski's model

\section{Non-symmetric frame}

Removing one of the springs, for example OC (Fig. 1), transforms the system into a non-symmetric one. The post-critical equilibrium state is shown in Fig. 2. It has bean constructed, using the free body diagram, where the spring $\mathrm{OA}$ has been replaced by the spring force $F_{1}$, so that the deformed system is acted upon by the forces $F_{1}$ and $P$. Moreover, the line of action of the spring force $F_{1}$ is defined by the points $\mathrm{A}$ and $\mathrm{O}$, while its intersection point $D$ with the vertical line through the point $\mathrm{B}$ of application of the load $P$ is the point of application of the resultant force $\vec{F}$ of the two forces, i. e. $\vec{F}=\vec{P}+\vec{F}_{1}$ (Fig. 2). In accordance with the elastic analogy [11], the line of action of $\vec{F}$ has to intersect the deformed column $\mathrm{OB}$, or its elastic extension at an inflexion 
point $\mathrm{T}$ of the elastic line. The inflection type of the elastic line is defined by the orientation of the resultant force $\vec{F}$. This very force is the resultant of the internal forces at the point of zero curvature, or the section with zero bending moment. There is a special case with $\vec{P}=-\vec{F}_{1}, \vec{F}=0$, where the elastic line is a circular arc of the radius $E I / M$. Here, $E$ is the modulus of elasticity, $I$ is the principal moment of inertia of the cross-section of the area $A$, and $M$ is the moment resultant of the couple $\vec{P}, \vec{F}_{1}$. Figure 2 represents the case, where the force of the spring OA is tensile, so that the force resultant $\vec{F}$ is located between $\vec{P}$ and $\vec{F}_{1}$. The lines of action of forces $\vec{P}, \vec{F}_{1}$ and $\vec{F}^{\prime}$ must share the same point $\mathrm{D}$, in accordance with the well-known three-force-body rule.

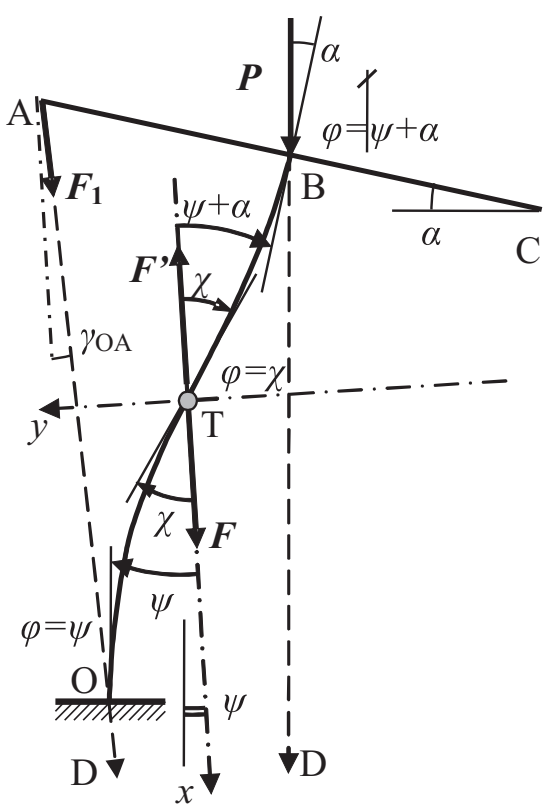

Fig. 2. First post-critical equilibrium state of the non-symmetric frame

Next, a rectangular coordinate system $x T y$ is adopted with $x$-axis oriented along the force-resultant $\vec{F}$ and $y$-axis, as shown in the Fig. 2. The axis $x$ is supposed tilted at an angle $\psi$ away from the vertical. Also, the angle made by the $x$-axis and the tangent line to the deformed column axis at section $\mathrm{T}$ is denoted by $\chi$ while $\varphi$ is the current angle from $x$-axis to the tangent line of an arbitrary section $\mathrm{S}$. The natural parameter for the elastic line is $s(\varphi)=T S$. The angle $\varphi$ itself varies in the interval $\psi \leq \varphi \leq \chi$, for the segment OT and in the interval $\psi+\alpha \leq \varphi \leq \chi$, for the segment TB, where $\alpha$ is the angle, formed by the beam AC and the horizontal line. With the coordinate system $x T y$ and 
the other parameters defined, we can write the Cartesian coordinates of the elastic line, as [11]:

$$
\begin{gathered}
x(\theta)=\sqrt{\frac{E I}{F}}\left[2 E\left(q^{2}\right)-2 E\left(q^{2}, \theta\right)-K\left(q^{2}\right)+F\left(q^{2}, \theta\right)\right], \\
y(\theta)=2 \sqrt{\frac{E I}{F}} q \cos \theta,
\end{gathered}
$$

and the natural parameter:

$$
s(\theta)=\sqrt{\frac{E I}{F}}\left[K\left(q^{2}\right)-F\left(q^{2}, \theta\right)\right] .
$$

Here, $K\left(q^{2}\right), F\left(q^{2}, \theta\right)$ are the complete and the incomplete elliptic integrals of the first kind, and $E\left(q^{2}\right), E\left(q^{2}, \theta\right)$ are the complete and the incomplete elliptic integrals of the second kind, respectively [12]. The following dimensionless parameters have been introduced in the above expressions for convenience:

$$
q=\sin \frac{\chi}{2}, \quad \theta=\arcsin \frac{\sin \frac{\varphi}{2}}{q}, \quad \Lambda=\sqrt{\frac{F L^{2}}{E I}} .
$$

It should be mentioned, that the relationships (1)-(4) are connected with the solution of the differential equation of the inextensible elastic line [1, 2, 6]: $\frac{d^{2} \varphi}{d s^{2}}-\frac{F}{E I} \sin \varphi=0$.

The spring force $F_{1}$ is determined by means of the elongation $\Delta l_{\mathrm{OA}}$ of the spring OA, caused by the deformation of the system (Fig. 2):

$$
\Delta l_{\mathrm{OA}}=\sqrt{\left(x_{\mathrm{O}}-x_{\mathrm{A}}\right)^{2}+\left(y_{\mathrm{O}}-y_{\mathrm{A}}\right)^{2}}-\sqrt{L^{2}+b^{2}} .
$$

Using (1) and (2) in (5), we get:

$$
\begin{aligned}
& x_{\mathrm{O}}=\sqrt{\frac{E I}{F}}\left[2 E\left(q^{2}\right)-2 E\left(q^{2}, \theta_{\mathrm{O}}\right)-K\left(q^{2}\right)+F\left(q^{2}, \theta_{\mathrm{O}}\right)\right], y_{\mathrm{O}}=2 \sqrt{\frac{E I}{F}} q \cos \theta_{\mathrm{O}}, \\
& x_{\mathrm{B}}=\sqrt{\frac{E I}{F}}\left[2 E\left(q^{2}\right)-2 E\left(q^{2}, \theta_{\mathrm{B}}\right)-K\left(q^{2}\right)+F\left(q^{2}, \theta_{\mathrm{B}}\right)\right], y_{\mathrm{B}}=2 \sqrt{\frac{E I}{F}} q \cos \theta_{\mathrm{B}} \\
& \theta_{\mathrm{O}}=\arcsin \frac{\sin \frac{\psi}{2}}{q}, \quad \theta_{\mathrm{B}}=\arcsin \frac{\sin \frac{\psi+\alpha}{2}}{q} \\
& x_{\mathrm{A}}=-x_{\mathrm{B}}-b \sin (\psi+\alpha), \quad y_{\mathrm{A}}=-y_{\mathrm{B}}+b \cos (\psi+\alpha) .
\end{aligned}
$$


If $c$ is the spring stiffness, then the spring force is:

$$
F_{1}=c \Delta l_{\mathrm{OA}}=c\left(\sqrt{\left(x_{\mathrm{O}}-x_{\mathrm{A}}\right)^{2}+\left(y_{\mathrm{O}}-y_{\mathrm{A}}\right)^{2}}-\sqrt{L^{2}+b^{2}}\right) .
$$

Next, we reduce the forces $\vec{P}$ and $\vec{F}_{1}$. For this purpose an angle:

$$
\gamma_{\mathrm{OA}}=\arctan \frac{y_{\mathrm{O}}-y_{\mathrm{A}}}{x_{\mathrm{O}}-x_{\mathrm{A}}},
$$

formed by $\vec{F}_{1}$ and $x$-axis is defined.

The reduction of the forces $\vec{P}$ and $\vec{F}_{1}$ yields:

$$
\begin{aligned}
& F_{x}=P_{x}+F_{1 x}=P \cos \psi+F_{1} \cos \gamma_{\mathrm{OA}}=F, \\
& F_{y}=P_{y}+F_{1 y}=-P \sin \psi+F_{1} \sin \gamma_{\mathrm{OA}}=0 .
\end{aligned}
$$

Let the force resultant $\vec{F}$ be applied at a certain point D. Apparently, it is the intersection point of the line $\overleftrightarrow{A O}$ and the vertical line through point $\mathrm{B}$, which is the line of action of the load $\mathrm{P}$. In order to define the position of D analytically, we first work out the equation of the straight line $\overleftrightarrow{A O}$ on given two points $\mathrm{A}$ and $\mathrm{O}$. With the help of expressions (6) and taking into account that the point $\mathrm{D}$ lies on $x$-axis with $y_{\mathrm{D}}=0$, we obtain:

$$
x_{\mathrm{D}}=\frac{x_{\mathrm{A}} y_{\mathrm{O}}-y_{\mathrm{A}} x_{\mathrm{O}}}{y_{\mathrm{O}}-y_{\mathrm{A}}}, \operatorname{tg} \psi=\frac{-y_{\mathrm{B}}}{x_{\mathrm{D}}-x_{\mathrm{B}}}=\frac{y_{\mathrm{B}}\left(y_{\mathrm{A}}-y_{\mathrm{O}}\right)}{\left(x_{\mathrm{A}}-x_{\mathrm{B}}\right) y_{\mathrm{O}}+\left(x_{\mathrm{B}}-x_{\mathrm{O}}\right) y_{\mathrm{A}}} .
$$

Neglecting the deformation of the column, due to the axial force, implies that the length of the column OB remains unchanged during deformation. In other words, the sum of arc lengths $O T$ and $T B$ has to be equal to $L$. Making use of (3), we have:

$$
\widehat{O T}=\sqrt{\frac{E I}{F}}\left[K\left(q^{2}\right)-F\left(q^{2}, \theta_{\mathrm{O}}\right)\right], \quad \stackrel{T B}{T B}=\sqrt{\frac{E I}{F}}\left[K\left(q^{2}\right)-F\left(q^{2}, \theta_{\mathrm{B}}\right)\right],
$$

so that:

$$
\sqrt{\frac{E I}{F}}\left[2 K\left(q^{2}\right)-F\left(q^{2}, \theta_{\mathrm{O}}\right)-F\left(q^{2}, \theta_{\mathrm{B}}\right)\right]=L
$$

Given the load $P$, geometry parameters of the frame and the material properties, the unknowns in the set of transcendental equations (9) - (12) are 
$\chi, \alpha, \psi$ and $F$. The following non-dimensional parameters are introduced for convenience:

$$
\bar{x}=x / L, \quad \bar{y}=y / L, \quad \Lambda_{1,2}=\sqrt{\frac{F_{1,2} L^{2}}{E I}}, \quad \Lambda_{p}=\sqrt{\frac{P L^{2}}{E I}} .
$$

After transformations from expressions (4) and (8)-(13), the following set of governing equations is arrived at:

$$
\begin{aligned}
& \Lambda_{p}^{2} \cos \psi+\Lambda_{1}^{2} \cos \gamma_{\mathrm{OA}}-\Lambda^{2}=0, \\
& -\Lambda_{p}^{2} \sin \psi+\Lambda_{1}^{2} \sin \gamma_{\mathrm{OA}}=0, \\
& {\left[\left(\bar{x}_{\mathrm{A}}-\bar{x}_{\mathrm{B}}\right) \bar{y}_{\mathrm{O}}+\left(\bar{x}_{\mathrm{B}}-\bar{x}_{\mathrm{O}}\right) \bar{y}_{\mathrm{A}}\right] \operatorname{tg} \psi+\bar{y}_{\mathrm{B}}\left(\bar{y}_{\mathrm{O}}-\bar{y}_{\mathrm{A}}\right)=0,} \\
& 2 K\left(q^{2}\right)-F\left(q^{2}, \theta_{\mathrm{O}}\right)-F\left(q^{2}, \theta_{\mathrm{B}}\right)-\Lambda=0 .
\end{aligned}
$$

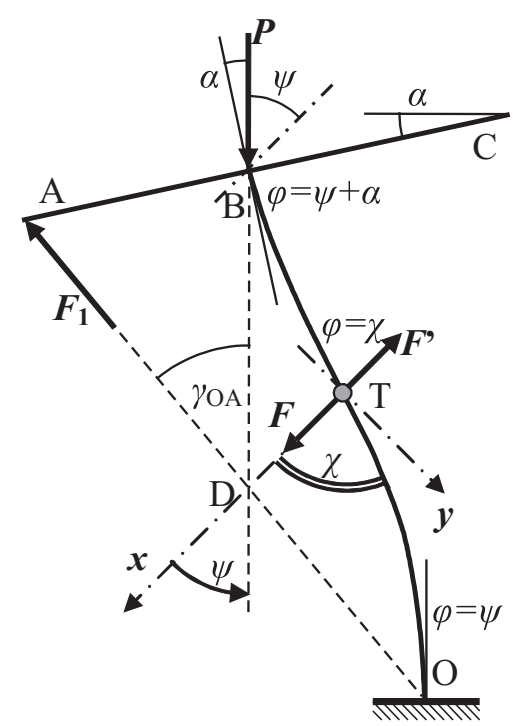

Fig. 3. Second post-critical equilibrium state of the non-symmetric frame

Figure 3 shows the second post-critical equilibrium configuration of the non-symmetric frame. It is characterized by the compression force in the spring OA. The corresponding set of governing equations is analogous to (14).

\section{Symmetric system}

The post-critical equilibrium configuration of the symmetric frame is shown in Fig. 4. The force $F_{2}$ in the second spring OC turns out to be com- 
pressive. Its inclusion in the set of governing equations necessitates working out expressions, analogous to (5), (6), (7) and (8), as follows:

$$
\begin{aligned}
& \Delta l_{\mathrm{OC}}=\sqrt{\left(x_{\mathrm{O}}-x_{\mathrm{C}}\right)^{2}+\left(y_{\mathrm{O}}-y_{\mathrm{C}}\right)^{2}}-\sqrt{L^{2}+b^{2}}, \\
& F_{2}=c \Delta l_{\mathrm{OC}}=c\left(\sqrt{\left(x_{\mathrm{O}}-x_{\mathrm{C}}\right)^{2}+\left(y_{\mathrm{O}}-y_{\mathrm{C}}\right)^{2}}-\sqrt{L^{2}+b^{2}}\right), \\
& \gamma_{\mathrm{OC}}=\operatorname{arctg} \frac{y_{\mathrm{O}}-y_{\mathrm{C}}}{x_{\mathrm{O}}-x_{\mathrm{C}}}, \\
& x_{\mathrm{C}}=-x_{\mathrm{B}}+b \sin (\psi+\alpha), \quad y_{\mathrm{C}}=-y_{\mathrm{B}}-b \cos (\psi+\alpha) .
\end{aligned}
$$

Thus, the terms $\Lambda_{2}^{2} \cos \gamma_{\mathrm{OC}}$ and $\Lambda_{2}^{2} \sin \gamma_{\mathrm{OC}}$ have to be added on the left hand sides of the first two equations (14), respectively. The fourth equation remains valid. In order to work out the third equation, we first define the force resultant $\vec{R}=R_{x} \vec{i}+R_{y} \vec{j}$ of the two spring forces $\vec{F}_{1}$ and $\vec{F}_{2}$ slid to the clamped end $\mathrm{O}$. The line of action of the force resultant $\vec{R}$ intersects the line of action of the load $P$ at a point $\mathrm{D}$, which evidently is the point of application of the force resultant $\vec{F}$ of the three forces $\vec{P}, \vec{F}_{1}$ and $\vec{F}_{2}$. The orientation of the force resultant $\vec{R}$ itself is defined by means of an angle $\gamma_{R}=\operatorname{arctg}\left(R_{y} / R_{x}\right)$. As for the location of the point $\mathrm{D}$, the coordinate $x_{\mathrm{D}}$ and its relationship with the angle $\psi$, we carry out the same procedure as in the previous section. The result is:

$$
x_{\mathrm{D}}=x_{\mathrm{O}}-y_{\mathrm{O}} \operatorname{cotg} \gamma_{R}, \operatorname{tg} \psi=\frac{-y_{\mathrm{B}}}{x_{\mathrm{D}}-x_{\mathrm{B}}}=\frac{y_{\mathrm{B}} \operatorname{tg} \gamma_{R}}{y_{\mathrm{O}}-\left(x_{\mathrm{O}}-x_{\mathrm{B}}\right) \operatorname{tg} \gamma_{R}} .
$$

In this way, the third equation reads:

$$
\left[\bar{y}_{\mathrm{O}}-\left(\bar{x}_{\mathrm{O}}-\bar{x}_{\mathrm{B}}\right) \operatorname{tg} \gamma_{R}\right] \operatorname{tg} \psi-\bar{y}_{\mathrm{B}} \operatorname{tg} \gamma_{R}=0 .
$$

The final form of the set of governing equations for the symmetric frame is:

$$
\begin{aligned}
& \Lambda_{p}^{2} \cos \psi+\Lambda_{1}^{2} \cos \gamma_{\mathrm{OA}}+\Lambda_{2}^{2} \cos \gamma_{\mathrm{OC}}-\Lambda^{2}=0, \\
& -\Lambda_{p}^{2} \sin \psi+\Lambda_{1}^{2} \sin \gamma_{\mathrm{OA}}+\Lambda_{2}^{2} \sin \gamma_{\mathrm{OC}}=0, \\
& {\left[\bar{y}_{\mathrm{O}}-\left(\bar{x}_{\mathrm{O}}-\bar{x}_{\mathrm{B}}\right) \operatorname{tg} \gamma_{R}\right] \operatorname{tg} \psi-\bar{y}_{\mathrm{B}} \operatorname{tg} \gamma_{R}=0,} \\
& 2 K\left(q^{2}\right)-F\left(q^{2}, \theta_{\mathrm{O}}\right)-F\left(q^{2}, \theta_{\mathrm{B}}\right)-\Lambda=0 .
\end{aligned}
$$




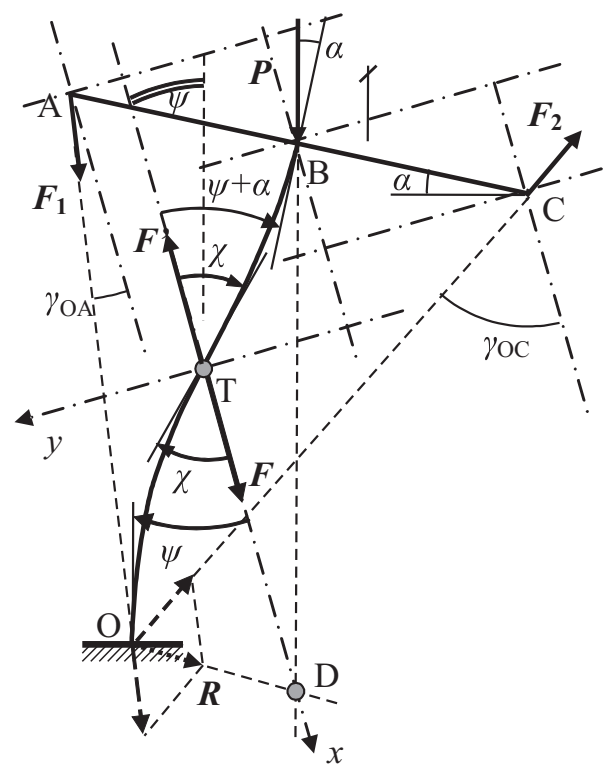

Fig. 4. Post-critical equilibrium state of the symmetric frame

4. Influence of the axial deformation over the critical and post-critical equilibrium state

In the paper [10], as well as in most of the investigations on critical and post-critical behaviour of deformable systems, the effect of the axial deformation due to normal forces is disregarded. The particularities of the frame under discussion raise a question whether and to what extent such an influence is related to the ratio between the spring constant $c$ and the specific axial column stiffness $E A / L$. With this end, in view the relationship:

$$
c=\zeta \frac{E A}{L}
$$

is introduced for convenience, where $\zeta$ is a constant and $A$ is the area of the column cross-section. The consequent analysis is similar to that of the preceding section for the symmetric frame, however, here the solution is related to the differential equation $\frac{d^{2} \varphi}{d s^{2}}-\frac{F}{E I}\left(1-\frac{F}{E A} \cos \varphi\right) \sin \varphi=0$ [7]. Thus, in lieu of 
the expressions $(1)-(3)$, we have:

(19) $x(z)=\frac{L \Omega}{\Lambda}\left\{\Psi\left[\Pi\left(n ; p^{2}, z\right)-\Pi\left(n, p^{2}\right)\right]+(1+\Psi)\left[K\left(p^{2}\right)-F\left(p^{2}, z\right)\right]\right\}$,

(20) $y(z)=\frac{2 L}{\Lambda} \sqrt{\frac{1+\varepsilon}{\varepsilon}} \operatorname{arctg}\left(q \sqrt{\frac{\varepsilon}{1+q^{2} \varepsilon}} \cos z\right)$,

(21) $s(z)=\frac{L \Omega}{\Lambda}\left[K\left(p^{2}\right)-F\left(p^{2}, z\right)\right]$.

New dimensionless parameters are utilized in (19)-(21), in addition to (4):

$$
i^{2}=\frac{I}{A}, \quad \lambda^{2}=\frac{L^{2}}{i^{2}}, \quad \frac{\Lambda^{2}}{\lambda^{2}}=\frac{\varepsilon}{1+\varepsilon}, \quad \varepsilon=\frac{\Lambda^{2}}{\lambda^{2}-\Lambda^{2}}, \quad p^{2}=q^{2} \frac{1+\varepsilon+q^{2} \varepsilon}{1+2 q^{2} \varepsilon},
$$

$$
\begin{aligned}
& z=\arcsin \left(\frac{1}{q} \sqrt{\frac{1+2 q^{2} \varepsilon}{1+\varepsilon\left(q^{2}+\sin ^{2} \frac{\varphi}{2}\right)}} \sin \frac{\varphi}{2}\right), \\
& \Psi=\frac{2\left(1+q^{2} \varepsilon\right)}{\varepsilon}, \Omega=\sqrt{\frac{1+\varepsilon}{1+2 q^{2} \varepsilon}}, n=\frac{q^{2} \varepsilon}{1+2 q^{2} \varepsilon} ; \\
& \Pi\left(n ; p^{2}\right)=\int_{0}^{\pi / 2} \frac{d z}{\left(1-n \sin ^{2} z\right) \sqrt{1-p^{2} \sin ^{2} z}} ; \text { and } \\
& \Pi\left(n ; p^{2}, z\right)=\int_{0}^{z} \frac{d z}{\left(1-n \sin ^{2} z\right) \sqrt{1-p^{2} \sin ^{2} z}}
\end{aligned}
$$

are the complete and incomplete elliptic integral of the third kind, respectively [12].

The coordinates of the characteristic points $\mathrm{O}$ and $\mathrm{B}$ of the frame are 
determined, as follows:

$$
\begin{aligned}
& z_{\mathrm{O}}=\arcsin \left(\frac{1}{q} \sqrt{\frac{1+2 q^{2} \varepsilon}{1+\varepsilon\left(q^{2}+\sin ^{2} \frac{\psi}{2}\right)}} \sin \frac{\psi}{2}\right), \\
& z_{\mathrm{B}}=\arcsin \left(\frac{1}{q} \sqrt{\frac{1+2 q^{2} \varepsilon}{1+\varepsilon\left(q^{2}+\sin ^{2} \frac{\psi+\alpha}{2}\right)}} \sin \frac{\psi+\alpha}{2}\right), \\
& \bar{x}_{\mathrm{O}}=\frac{\Omega}{\Lambda}\left\{\Psi\left[\Pi\left(n ; p^{2}, z_{\mathrm{O}}\right)-\Pi\left(n, p^{2}\right)\right]+(1+\Psi)\left[K\left(p^{2}\right)-F\left(p^{2}, z_{\mathrm{O}}\right)\right]\right\}, \\
& \bar{y}_{\mathrm{O}}=\frac{2}{\Lambda} \sqrt{\frac{1+\varepsilon}{\varepsilon}} \operatorname{arctg}\left(q \sqrt{\frac{\varepsilon}{1+q^{2} \varepsilon}} \cos z_{\mathrm{O}}\right), \\
& \bar{x}_{\mathrm{B}}=\frac{\Omega}{\Lambda}\left\{\Psi\left[\Pi\left(n ; p^{2}, z_{\mathrm{B}}\right)-\Pi\left(n, p^{2}\right)\right]+(1+\Psi)\left[K\left(p^{2}\right)-F\left(p^{2}, z_{\mathrm{B}}\right)\right]\right\}, \\
& \bar{y}_{\mathrm{B}}=\frac{2}{\Lambda} \sqrt{\frac{1+\varepsilon}{\varepsilon}} \operatorname{arctg}\left(q \sqrt{\frac{\varepsilon}{1+q^{2} \varepsilon}} \cos z_{\mathrm{B}}\right), \\
& \widetilde{O T}=\frac{L \Omega}{\Lambda}\left[K\left(p^{2}\right)-F\left(p^{2}, z_{\mathrm{O}}\right)\right], \quad \overparen{T B}=\frac{L \Omega}{\Lambda}\left[K\left(p^{2}\right)-F\left(p^{2}, z_{\mathrm{B}}\right)\right] .
\end{aligned}
$$

The governing equations here possess the same physical meaning as in the preceding sections 2 and 3. The fourth equation, however, has to take into account the elastic shortening of the column. For clarity, let us consider a differentially small segment $d s$ at a distance $s$ from the inflexion point $\mathrm{T}$ to the clamped end $\mathrm{O}$. The related normal force is equal to $F \cos \varphi$, while the elastic shortening of $d s$ is $F d s \cos \varphi /(E A)$. The total shortening of the entire segment $\widetilde{O T}$ of length $l$ prior to deformation is:

$$
\Delta_{\overparen{T O}}=\int_{0}^{l} \frac{F \cos \varphi}{E A} d s=\int_{0}^{x_{\mathrm{O}}} \frac{F}{E A} d x=\frac{F x_{\mathrm{O}}}{E A},
$$

and that for the remaining segment TB of length $L-l$ prior to deformation is

$$
\Delta_{\overparen{T B}}=\int_{0}^{L-l} \frac{F \cos \varphi}{E A} d s=\int_{0}^{x_{\mathrm{B}}} \frac{F}{E A} d x=\frac{F x_{\mathrm{B}}}{E A} .
$$

In this way, the fourth equation has the form $\overparen{O T}+\overparen{T B}=L-\Delta_{\overparen{T B}}-$ 
$\Delta_{\widehat{O T}}$. This is further transformed with the aid of expressions $(22)-(25)$, to give:

$$
\begin{aligned}
& \frac{\Omega}{1+\varepsilon}\left\{2\left(1+q^{2} \varepsilon\right)\left[\Pi\left(n ; p^{2}, z_{\mathrm{O}}\right)+\Pi\left(n ; p^{2}, z_{\mathrm{B}}\right)-2 \Pi\left(n, p^{2}\right)\right]+\right. \\
& \left.+\left(3+2 \varepsilon+2 q^{2} \varepsilon\right)\left[2 K\left(p^{2}\right)-F\left(p^{2}, z_{\mathrm{O}}\right)-F\left(p^{2}, z_{\mathrm{B}}\right)\right]\right\}-\Lambda=0,
\end{aligned}
$$

The final form of the remaining three equations is:

$$
\begin{aligned}
& \Lambda_{p}^{2} \cos \psi+\Lambda_{1}^{2} \cos \gamma_{\mathrm{OA}}+\Lambda_{2}^{2} \cos \gamma_{\mathrm{OC}}-\Lambda^{2}=0, \\
& \Lambda_{p}^{2} \sin \psi+\Lambda_{1}^{2} \sin \gamma_{\mathrm{OA}}+\Lambda_{2}^{2} \sin \gamma_{\mathrm{OC}}=0, \\
& {\left[\bar{y}_{\mathrm{O}}-\left(\bar{x}_{\mathrm{O}}-\bar{x}_{\mathrm{B}}\right) \operatorname{tg} \gamma_{R}\right] \operatorname{tg} \psi-\bar{y}_{\mathrm{B}} \operatorname{tg} \gamma_{R}=0 .}
\end{aligned}
$$

\section{Numerical solutions}

Consider a nonsymmetrical frame (Figs 2 and 3$)$ with $b=L / 2(\beta=1 / 2)$ and $\zeta L^{2} / i^{2}=100$. Table 1 shows some numerical results for the set of equations (14), obtained by means of MATLAB software. Given the loading parameter $\Lambda_{p}$, the unknowns $\chi, \psi, \alpha$ and $\Lambda$ are obtained first and $\Lambda_{1}$ afterwards. From the last column of the same table it is seen, that the critical parameter $\Lambda_{p, c r}$ tends to 3.34. As might have been expected, the spring has a stabilizing effect on the frame. Otherwise, without it the critical parameter would have been $\Lambda_{p, c r}^{2}=$ $\pi^{2} / 4 \approx 2.47$, as in the classical Euler's case of cantilevered column. The upper three lines refer to the second post-critical configuration of the non-symmetric frame (Fig. 3). The negative sign for the angle $\chi$ has been introduced for the sake of convenience in construction in Fig. 5. On the same reason, a negative sign has been assumed for the spring force parameter $\Lambda_{1}^{2}$. The spring $\mathrm{OA}$ is compressed in the second post-critical configuration, stretched in the first post-critical configuration and unstrained with the loading parameter $\Lambda_{p}$, approaching its critical value.

It is worthwhile noting, that the loading parameter $\Lambda_{p}$ decreases, when approaching the critical point in the left half-plane, by the second post-critical configuration and keeps decreasing away from the critical point in the right half-plane, by the first post-critical configuration. Simultaneously, the force $F$ in the column, represented by the parameter $\Lambda^{2}$, increases from left to right by the second and the first post-critical configuration, respectively. The explanation about the second post-critical form lies in a relatively weaker assistance, the force $F^{\prime}$ receives in equilibrating the load $P$ from steadily decreasing, by absolute value spring force $F_{1}$. 
Table 1. Results obtained from set of equations (14)

\begin{tabular}{||cccccc||}
\hline \hline$\chi$ & $\psi$ & \multicolumn{1}{c}{$\alpha$} & \multicolumn{1}{c}{$\Lambda^{2}$} & \multicolumn{1}{c}{$\Lambda_{1}^{2}$} & \multicolumn{1}{c}{$\Lambda_{p}^{2}$} \\
\hline \hline-0.402 & 0.1553 & 0.1932 & 2.94 & -0.75 & 3.50 \\
-0.150 & 0.0492 & 0.0797 & 3.15 & -0.30 & 3.40 \\
-0.011 & 0.0032 & 0.0063 & 3.33 & -0.02 & 3.35 \\
\hline 0.004 & 0.0013 & 0.0026 & 3.35 & 0.00 & 3.34 \\
\hline 0.110 & 0.0269 & 0.0665 & 3.52 & 0.06 & 3.30 \\
\hline 0.247 & 0.0469 & 0.1625 & 3.80 & 0.33 & 3.25 \\
\cline { 1 - 4 } 0.322 & 0.0504 & 0.2214 & 3.98 & 0.60 & 3.23 \\
\hline 0.405 & 0.0474 & 0.2934 & 4.19 & 1.01 & 3.20 \\
\hline \hline
\end{tabular}

The growth of the internal force $F$, on the other hand, is due to the relatively stronger increase of the force in the spring OA, than the reduction of the load $P$. This description corresponds to the variations of the graphs for the parameters $\Lambda^{2}, \Lambda_{1}^{2}$ and $\Lambda_{p}^{2}$ in Fig. 5, for the post-critical equilibrium state of the frame, depending on the rotation angle $\chi$ of the section $\mathrm{T}$ at the point of inflexion of the elastic line.

Figure 6 shows variations of some frame parameters, depending on the column axial stiffness constant $\zeta$, the constant loading parameter $\Lambda_{p}^{2}=3.2$ and the first post-critical equilibrium configuration. The values for the angle $\psi$ have been multiplied by 10 for better visualization. On the same reason, $\Lambda$ and $\Lambda_{1}$ have been plotted in place of $\Lambda^{2}$ and $\Lambda_{1}^{2}$. As one might expect, the forces in the column $(\Lambda)$ and in the spring $\left(\Lambda_{1}\right)$ increase, when the stiffness constant $\zeta$ is increased.

The numerical analysis for the symmetric frame (Fig. 4) is based on the set of transcendental equations (17). Figure 7 shows the variation of the force parameters $\Lambda^{2}, \Lambda_{p}^{2}, \Lambda_{1}^{2}$ and $\Lambda_{2}^{2}$, depending on the rotation angle $\alpha$ of the upper column section for $\zeta L^{2} / i^{2}=100$. It is seen, that the spring force $F_{1}$ is tensile for small values of $\alpha$, then attains a maximum, decreases to zero for $\alpha \approx 0.34$ and becomes compressive afterwards. On the contrary, the spring force $F_{2}$ is compressive and increases by absolute value steadily with increasing angle $\alpha$. Simultaneously, the loading parameter $\Lambda_{p}$ increases with increasing angle $\alpha$, as is the case with a stable critical point. On the other hand, it is observed that the effect of the second spring on the critical value of the loading is small in size. While the critical parameter by one spring is $\Lambda_{p, c r}^{2} \approx 3.34$, by two springs 


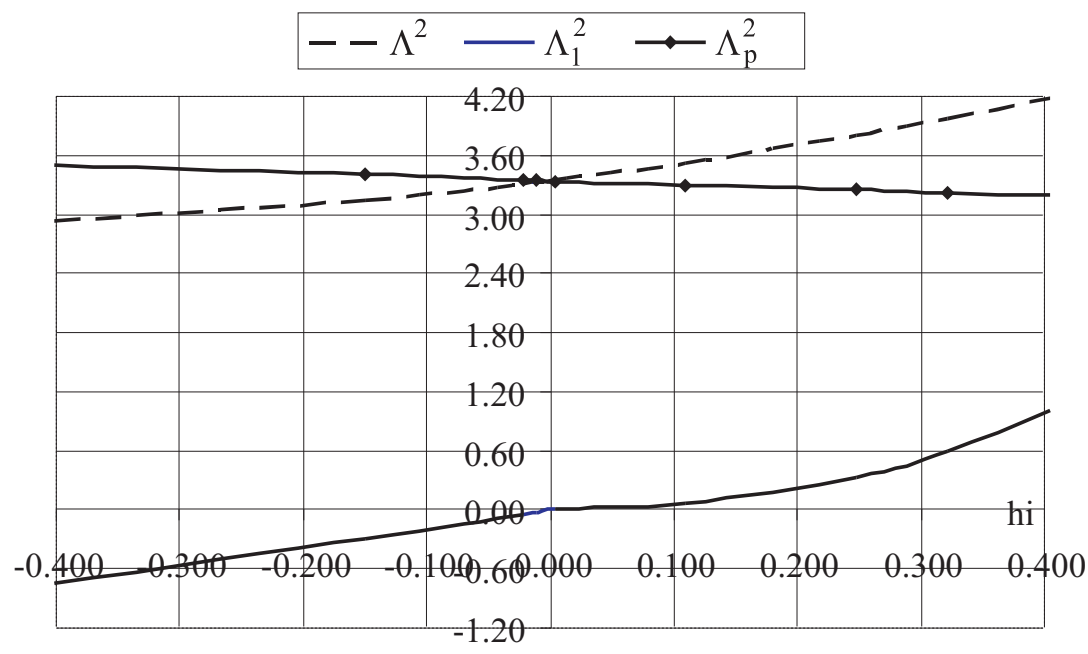

Fig. 5. Variation of $\Lambda^{2}, \Lambda_{1}^{2}$ and $\Lambda_{p}^{2}$ vs. $\chi$ for $\zeta L^{2} / i^{2}=100$

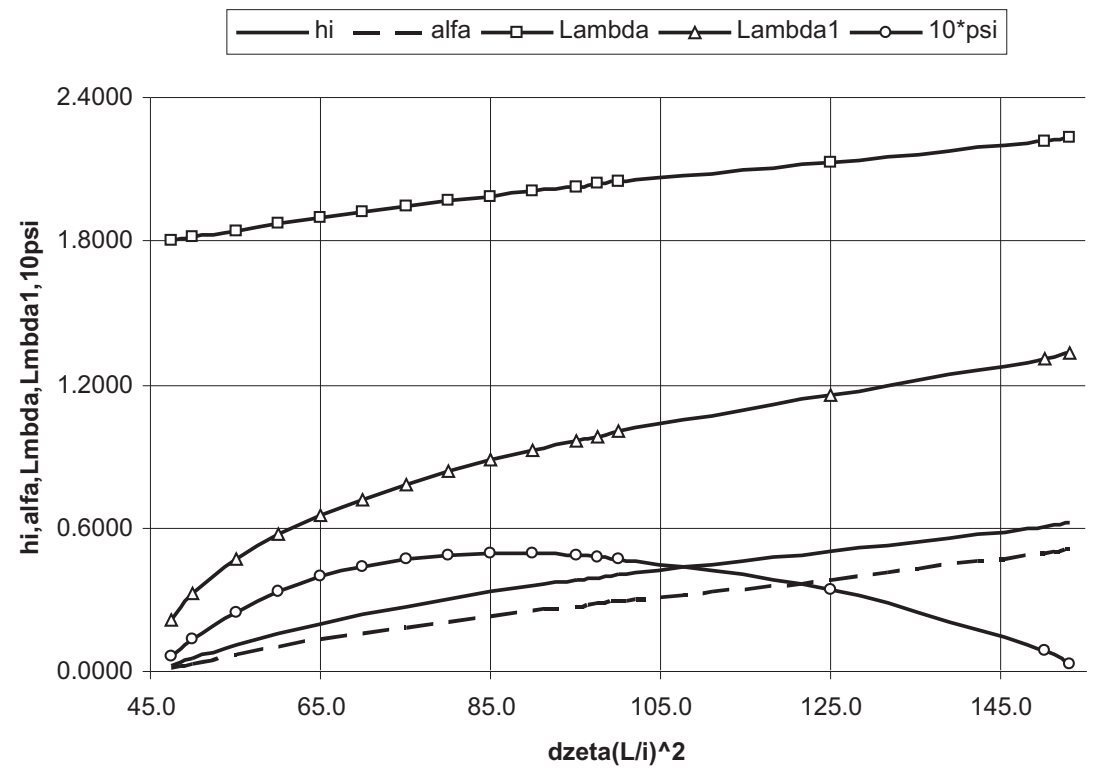

Fig. 6. Variation of $\chi, \alpha, \Lambda, \Lambda_{1}$ and $10 \psi$ for $\Lambda_{p}^{2}=3.2$ depending on $\zeta L^{2} / i^{2}$

it is $\Lambda_{p, c r}^{2} \approx 3.41$. The presence of the second spring, however, leads to decrease of the internal force $F$ in the column in comparison to its critical value. This 


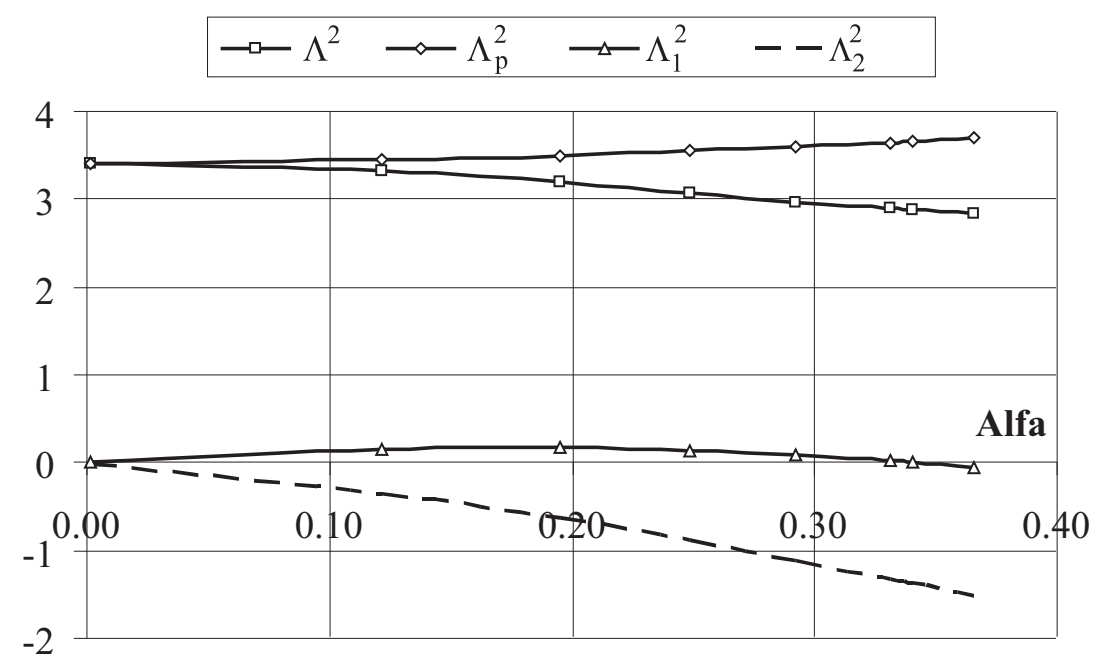

Fig. 7. Variation of $\Lambda^{2} \Lambda_{p}^{2}, \Lambda_{1}^{2}$ and $\Lambda_{2}^{2}$ vs. $\alpha$ for $\zeta L^{2} / i^{2}=100$

fact, particularity of the post-critical behaviour, is illustrated by the graph for the parameter $\Lambda^{2}$ in Fig. 7 .

Table 2 shows variation of the symmetric frame parameters, depending on the beam $\mathrm{AC}$ length $2 b$, which is varied from $0.3 L$ to $L$. The numerical results have been obtained for loading parameter $\Lambda_{p}^{2}=3.5$ and spring stiffness parameter $\zeta L^{2} / i^{2}=100$. It is seen, that the column force $F$ (or $\Lambda^{2}$ ) grows, when the length of the beam is increased, while the force in the compression spring $\left(\Lambda_{2}^{2}\right)$ decreases by absolute value. The force in the tension spring $\left(\Lambda_{1}^{2}\right)$, on the other hand, increases first, attains a maximum for $\beta=0.25$ and decreases afterwards (cf. Fig. 8).

As is the case with the column force, the critical parameter of the load $\Lambda_{p, c r}^{2}$ also increases with increasing $\beta$, albeit rather slightly. For example, for $\beta=0.3$ we obtain: $\Lambda_{p, c r}^{2}=3.317$, for $\beta=0.4$ the result is $\Lambda_{p, c r}^{2}=3.38$, etc.

The set of transcendental equations (26), (27) has been solved numerically, in order to analyze the influence of the axial deformation of the column upon the critical and the post-critical system behaviour. Table 3 contains some numerical results for the frame with $(\varepsilon>0)$ and without $(\varepsilon=0)$ axial deformations by different spring constants and constant loading parameter $\Lambda_{p}^{2}=3.5$. The last column of the table shows the percentage difference between the parameters $\Lambda$ of the column force $F$ for extensible and inextensible case, respectively. It appears that the influence of the axial deformation of the 
Table 2. Variation of the symmetric frame parameters vs $\beta$ for $\zeta L^{2} / i^{2}=100$ in post-critical equilibrium state

\begin{tabular}{|c|c|c|c|c|c|c|}
\hline$\beta=b / L$ & $\chi$ & $\psi$ & $\alpha$ & $\Lambda^{2}$ & $\Lambda_{1}^{2}$ & $\Lambda_{2}^{2}$ \\
\hline 0.15 & 1.2827 & 0.7481 & 0.4867 & 1.3927 & 0.1810 & -2.6340 \\
\hline 0.2 & 0.9367 & 0.4738 & 0.4011 & 1.5024 & 0.2927 & -2.0788 \\
\hline 0.25 & 0.7372 & 0.3323 & 0.3380 & 1.5956 & 0.2974 & -1.6277 \\
\hline 0.3 & 0.6127 & 0.2536 & 0.2918 & 1.6625 & 0.2712 & -1.2927 \\
\hline 0.35 & 0.5286 & 0.2055 & 0.2575 & 1.7096 & 0.2404 & -1.0486 \\
\hline 0.4 & 0.4685 & 0.1739 & 0.2314 & 1.7431 & 0.2126 & -0.8695 \\
\hline 0.45 & 0.4237 & 0.1520 & 0.2113 & 1.7676 & 0.1895 & -0.7361 \\
\hline 0.5 & 0.3892 & 0.1360 & 0.1953 & 1.7859 & 0.1707 & -0.6349 \\
\hline
\end{tabular}

column on the column force $F$ is small. The same conclusion holds for the corresponding critical parameter $\Lambda_{p, c r}$.

Table 3. Some results, derived from equations (26), (27)

\begin{tabular}{|c|cccccc|}
\hline$\zeta L^{2} / i^{2}$ & $\varepsilon$ & $\chi$ & $\psi$ & $\alpha$ & $\Lambda$ & $\%$ \\
\hline \multirow{2}{*}{100} & $>0$ & 0.3542 & 0.01223 & 0.1788 & 1.7915 & \multirow{2}{*}{0.31} \\
& $=0$ & 0.3892 & 0.136 & 0.1953 & 1.7859 & \\
\hline \multirow{2}{*}{150} & $>0$ & 0.2178 & 0.0737 & 0.1094 & 1.8059 & \multirow{2}{*}{0.25} \\
& $=0$ & 0.2705 & 0.0924 & 0.1353 & 1.8014 & \\
\hline \multirow{2}{*}{175} & $>0$ & 0.1836 & 0.06104 & 0.09263 & 1.8174 & \multirow{2}{*}{0.49} \\
& $=0$ & 0.2332 & 0.0789 & 0.1167 & 1.8085 & \\
\hline \multirow{2}{*}{181.5} & $>0$ & 0.16883 & 0.05612 & 0.08511 & 1.8177 & \multirow{2}{*}{0.41} \\
& $=0$ & 0.22494 & 0.0759 & 0.11262 & 1.8102 & \\
\hline \multirow{2}{*}{200} & $>0$ & 0.15646 & 0.0513 & 0.07919 & 1.8265 & \multirow{2}{*}{0.65} \\
& $=0$ & 0.2041 & 0.0684 & 0.1023 & 1.8147 & \\
\hline
\end{tabular}

The effect of the axial deformation of the column appears more pronounced on the displacements of the post-critical equilibrium configuration. Figure 9 depicts post-critical equilibrium forms for two values of the spring parameter $\zeta L^{2} / i^{2},=100$ and 200 , respectively, constant loading parameter $\Lambda_{p}^{2}=3.5$ both with and without axial deformations. It was found, that the deflection of the upper end of the inextensible column is greater, than that of the extensible one. Moreover, increase in the spring stiffness results in column deflections decrease. 


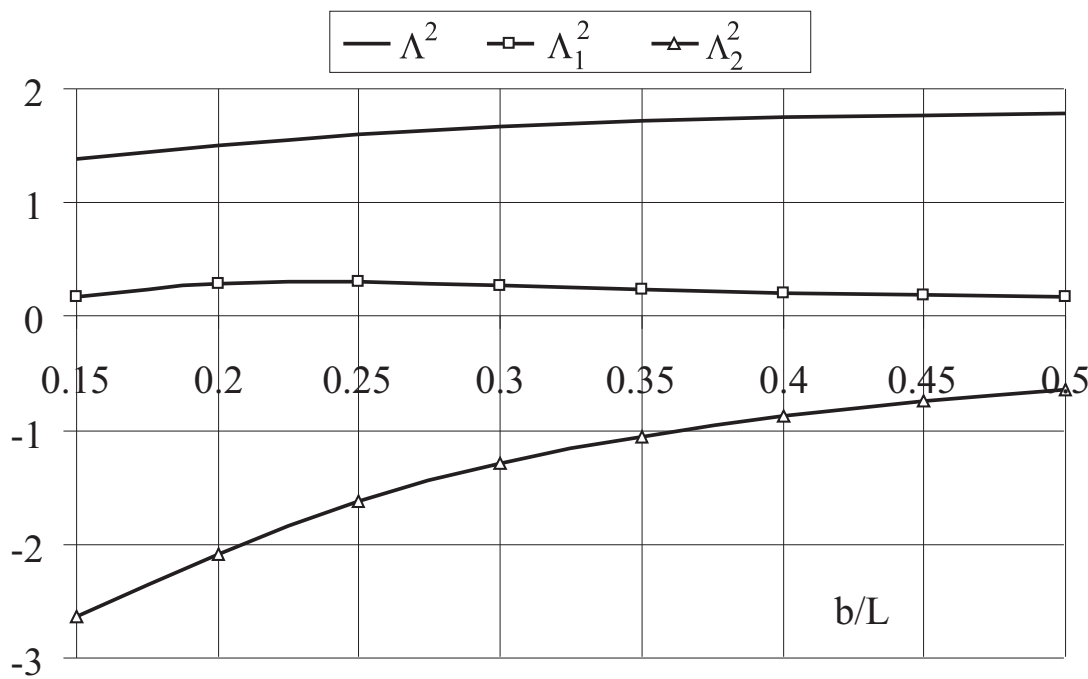

Fig. 8. Variation of $\Lambda^{2}, \Lambda_{1}^{2}$ and $\Lambda_{2}^{2}$ vs $\beta$ for $\Lambda_{p}^{2}=3.5$ and $\zeta L^{2} / i^{2}=100$, by post-critical equilibrium state

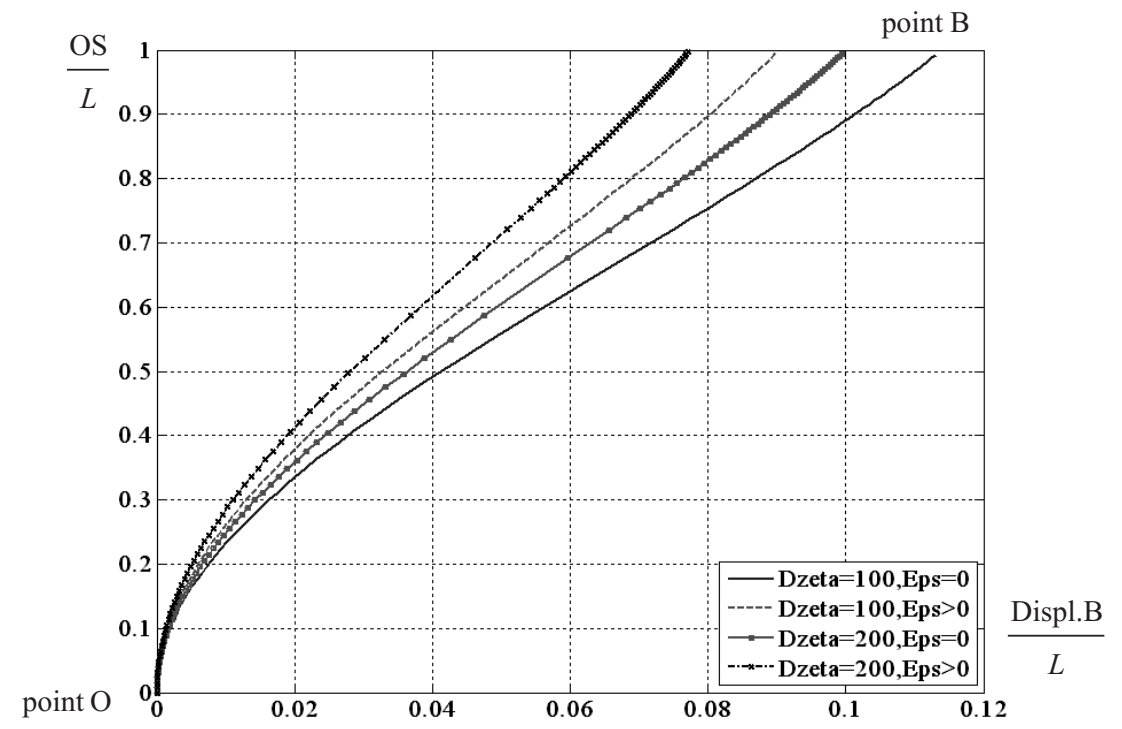

Fig. 9. Post-critical equilibrium forms of the column for $\zeta L^{2} / i^{2}=100$ and 200

\section{Conclusions}

Critical and post-critical analysis of a T-shaped frame, strengthened 
with two linear springs, proposed and analyzed previously by Życzkowski [10], has been considered in the present paper. The solution is exact, making use of the Euler elastic approach and a frame of reference originated in the point of inflexion of the column axis. Numerical results, obtained with the help of MATLAB software package, show the effect of the strengthening springs on the critical and post-critical system behaviour. The post-critical equilibrium forms, encountered during the investigation, appeared as double curvature lines, which means that the conclusions of [10], based on a single curvature line, are not so correct and we must be attentive, when we using them. The springs stabilize the column in the sense that they lead to an increase of the critical load. The influence is relatively stronger in the case of one sided strengthening, whereas, a two sided strengthening results in decrease in the column internal force. The beam length also exerts a strong influence on the critical and postcritical frame stressed and strained state. Increasing the beam length results in increasing column internal force along with the critical load, whereas the force in the compressed spring decreases by absolute value. In contrast, the force in the stretched spring increases first, attains a maximum value and decreases afterwards. The effect of the extensibility features of the column on its critical and post-critical behaviour has been studied, too. It turns out to be small, as far as the critical load and column force are concerned and more noticeable with the deflections of the movable end of the column.

\section{REFERENCES}

[1] Popov, E. P. Nonlinear Problems of Statics of Thin Rods, Leningrad, Moscow, Gostekhizdat, 1948 (in Russian).

[2] Britvec, S. J. The Stability of Elastic Systems, New York, Pergamon press, 1973.

[3] Leipholz, H. Stabilität Elastischer Systeme, Karlsruhe, G. Braun, 1960.

[4] Volimir, A. S. Stability of Deformable Systems, Moscow, Nauka, 1963 (in Russian).

[5] Bazant, Z. P., L. Cedolin. Stability of Structures: Elastic, Inelastic, Fracture and Damage Theories, New Jersey, World Scientific, 2010.

[6] Burgermeitster, G., H. Steup, H. Kretzschmar. Stabilitätstheorie, Berlin, Akademie-Verlag, Teil 1, 1966.

[7] Pfluger, A. Stabilitätsprobleme der Elastostatik, Berlin, Springer, 1950.

[8] Chen, W.-F., T. Atsuta. Theory of Beam-columns, Vol. 1: In-plane Behaviour and Design, New York, Mc Graw-Hill, 1981. 
[9] Sashrov, Yu. L., S. V. Levyakov. Stability of Inflectional Elasticae Centered at Vertices or Inflection Points. Proceedings of the Steklov Institute of Mathematics, 271 (2010), 177-192.

[10] ŻYCZKowski, M. Post-buckling Analysis of Non-prismatic Columns under General Behaviour of Loading. International Journal of Non-Linear Mechanics. 40 (2005), 445-463.

[11] Mladenov, K. Elastic Analogy Rule, Annual of the University of Architecture, Civil Engineering and Geodesy - Sofia, Vol. XXXII (1985-1986), No. 5, 76-84 (in Bulgarian).

[12] Abramovitz, M., I. A. Stegun (Eds.). Handbook of Mathematical Functions with Formulas, Graphs and Mathematical Tables, National Bureau of Standards, 1964. 